\title{
CORREÇÃO DE DADOS DE TEMPERATURA DE FUNDO DE POÇO (TFP)
}

\author{
Anderson Gusmão Cavalcante ${ }^{1}$, Roberto Max de Argollo² e Humberto da Silva Carvalho $^{3}$ \\ Recebido em 9 novembro, 2004 / Aceito em 28 dezembro, 2004 \\ Received November 9, 2004 / Accepted December 28, 2004
}

\begin{abstract}
BHT) from 207 wells distributed throughout the Recôncavo, Camamu, Almada, Cumuruxatiba and Jequitinhonha sedimentary basins, Bahia-Brazil, were corrected to obtain true temperatures of the formations. The methodology used to correct these temperatures consisted in using the Eq.(1) numerically resolved as the data set shows two or more registers of temperatures at the same depth (exponential integral method) and in application of the time-depth scheme as we had only one temperature registered per depth.

The corrections obtained by the two methods show that the formation static temperatures are 5 to $20^{\circ} \mathrm{C}$ greater than the non-corrected ones. We tested the validity of the time-depth scheme applying the empirical equation developed in this work on the multiple temperature registers taking the higher ones among them. The average geothermal gradient within the basins, obtained with the temperatures corrected by that method, presents a discrepancy of only 2,8\% as compared to the one obtained with the corrected data by the exponential integral method, shows that it is a valid method to correct temperatures in the basins studied.
\end{abstract}

The mean geothermal gradient obtained with corrected BHTs within all basins was $(27,9 \pm 2,1) \times 10^{-3 \circ} \mathrm{C} \mathrm{m}^{-1}$.

Keywords: bottom-hole temperature, BHT correction, geothermal gradient.

RESUMO. 577 medidas de temperatura de fundo de poço (TFP) oriundas de 207 poços distribuídos pelas bacias sedimentares do Recôncavo, Camamu, Almada, Cumuruxatiba e Jequitinhonha, Bahia, Brasil, foram corrigidas para obter-se as temperaturas estáticas das respectivas formações. A metodologia utilizada para correção destas temperaturas consistiu no uso da Eq.(1) resolvida numericamente quando os dados apresentavam dois ou mais registros de temperatura numa mesma profundidade (método das integrais exponenciais) e na aplicação do esquema de tempo-profundidade quando tivemos apenas um registro de temperatura numa dada profundidade.

As correções obtidas pelos dois métodos mostram que as temperaturas estáticas da formação são 5 a $20^{\circ} \mathrm{C}$ maiores que as temperaturas não-corrigidas. Testamos a validade do esquema tempo-profundidade aplicando a equação empírica desenvolvida neste trabalho nas medidas de múltiplas temperaturas, tomando-se a maior temperatura entre as múltiplas. 0 gradiente geotérmico médio obtido com as temperaturas corrigidas por este método apresenta uma discrepância de apenas 2,8\% quando comparado com o gradiente obtido com os dados corrigidos pelo método das integrais exponenciais, validando, assim, 0 uso daquele esquema nas bacias estudadas.

0 gradiente geotérmico médio obtido com as TFPs corrigidas em toda a área estudada foi de $(27,9 \pm 2,1) \times 10^{-3 \circ} \mathrm{C} \mathrm{m}^{-1}$.

Palavras-chave: temperatura de fundo de poço, correção de TFP, gradiente geotérmico.

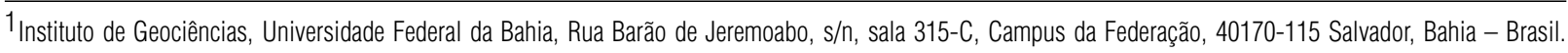
Tel: (71) 3203-8544; Fax: (71) 3203-8501 - E-mail: agc@cpgg.ufba.br

2Universidade Federal da Bahia, Instituto de Física, Centro de Pesquisa em Geofísica e Geologia, Laboratório de Física Nuclear Aplicada, Travessa Barão de Jeremoabo, s/n, Campus da Federação 40170-280 Salvador, Bahia - Brasil. Tel: (71) 3263-6680; Fax: (71) 3235-0002 - E-mail: robmax@ufba.br

3 Universidade Federal da Bahia, Instituto de Física, Centro de Pesquisa em Geofísica e Geologia, Laboratório de Física Nuclear Aplicada, Travessa Barão de Jeremoabo, s/n, Campus da Federação, 40170-280 Salvador, Bahia - Brasil. Tel: (71) 3263-6680; Fax: (71) 3235-0002 - E-mail: dgn@ufba.br
} 


\section{INTRODUÇ̃̃o}

As bacias sedimentares, onde a atividade exploratória de petróleo constitui um dos principais interesses, é uma fonte importante de informações para estudos geotérmicos. A temperatura é reconhecidamente um dos agentes mais importantes na maturação de hidrocarbonetos que controla os processos de formação e destruição de óleo e gás (Carvalho et al., 1987). Contudo, as temperaturas de fundo de poço, registradas durante as operações de perfilagem, apresentam valores sempre menores que as verdadeiras temperaturas estáticas da formação, devido, sobretudo, aos processos de perfuração do poço e de circulação da lama que causam perturbações na temperatura da formação em torno do poço. Para a determinação do fluxo de calor geotérmico, ou para calibração de modelos termomecânicos utilizados na avaliação de reservatórios, é desejável conhecer-se a temperatura estática da formação com um alto grau de confiança. A precisão e acurácia associadas à medida da temperatura corrigida dependem da precisão e acurácia com que são conhecidas as medidas referidas acima e do método utilizado no tratamento dessas medidas (Cavalcante, 2004), como também da eliminação de possíveis erros sistemáticos existentes no próprio modelo.

As temperaturas registradas durante as operações de perfilagem são afetadas pela circulação do fluído de perfuração, podendo atingir valores de até $40^{\circ} \mathrm{C}$ inferiores à verdadeira temperatura estática da formação no momento em que a circulação é cessada (Raymond, 1969). Já segundo Deming (1989), as TFPs são geralmente mais baixas do que a temperatura verdadeira da formação por um valor de 10 a $15^{\circ} \mathrm{C}$ em média, mas provavelmente varia de área para área. Este efeito de esfriamento é particularmente observado em poços perfurados na plataforma continental de espessa lâmina d'água, nos quais, a água do mar mais fria, refrigera o fluído de perfuração que, conseqüentemente, retira mais calor dos poços do que em outras condições.

Diversos fatores contribuem para perturbar a temperatura verdadeira da formação como, por exemplo, as características mecânicas do poço (diâmetro), a duração da perfuração, a troca de calor entre poço e formação, as propriedades térmicas do fluído de perfuração e das rochas adjacentes, o fluxo de fluídos na formação, etc. 0 tempo necessário para o poço recuperar o calor dissipado pela circulação e atingir a temperatura de equilíbrio é normalmente muitas horas superior ao tempo gasto nas operações de perfuração (Bullard, 1947).

Estes tempos, demasiadamente longos, inviabilizam a utilização direta de dados de TFP, uma vez que, na maioria dos casos, as medidas são feitas antes da estabilização térmica do poço. Por esta razão, vários métodos de correção de temperatura foram desenvolvidos ao longo do tempo, com a finalidade de estimar a verdadeira temperatura estática da formação utilizando as temperaturas medidas de fundo de poço, possibilitando, assim, 0 uso desses dados em estudos geotérmicos.

Esses métodos dividem-se em duas classes de modelos, que simulam os distúrbios térmicos devidos à perfuração e circulação da lama, como também à recuperação térmica subsequente. Uma classe tenta simular a evolução da temperatura em toda a coluna de lama e necessita de conhecimentos detalhados da história da perfuração, tais como a composição da lama, temperatura da lama injetada, taxa de circulação do fluído e propriedades físicas das rochas. A outra classe concentra-se na região do fundo do poço, onde as temperaturas são rotineiramente medidas durante a perfilagem. Para uma descrição completa dos modelos referentes às duas classes mencionadas, 0 leitor poderá consultar Deming (1989) e Cavalcante (2004).

Neste trabalho desenvolvemos metodologias para corrigir 577 dados de temperaturas de fundo de poço de cerca de 207 poços distribuídos pelas bacias sedimentares do Recôncavo, Camamu, Almada, Cumuruxatiba e Jequitinhonha, todas localizadas no estado da Bahia, Brasil.

\section{METODOLOGIA}

\section{Revisão teórica}

Em seu clássico artigo, Bullard (1947) descreve o comportamento da evolução da temperatura dentro de um poço perfurado num meio com propriedades térmicas homogêneas. Lachenbuch \& Brewer (1959), baseados no modelo de uma fonte linear de calor devido a Bullard (1947), apresentaram uma expressão relativamente simples para descrever a evolução da temperatura no poço após os períodos de perturbação. A solução para este problema é dada pela expressão

$$
\begin{aligned}
T\left(t_{d}\right)=T_{\infty} & +\frac{Q}{4 \pi K}\left\{E_{i}\left[-\frac{r^{2}}{4 \kappa\left(t_{d}+t_{c}\right)}\right]\right. \\
& \left.-E_{i}\left(-\frac{r^{2}}{4 \kappa t_{d}}\right)\right\},
\end{aligned}
$$

onde

$\mathrm{T}_{\infty}$ : temperatura estática da formaçã̃o (TEF);

$\mathrm{T}\left(\mathrm{t}_{d}\right)$ : temperatura medida no fundo do poço;

$\mathrm{t}_{c}$ : tempo de circulação da lama;

$\mathrm{t}_{d}$ : tempo decorrido desde 0 término da circulação da lama e 0 instante em que foi feita a medida da temperatura (tempo de descanso do poço); 
Q: taxa na qual calor é suprido $\left(\mathrm{W} \mathrm{m}^{-1}\right)$;

$\mathrm{K}$ : condutividade térmica do sistema composto pela lama do poço e a formação;

$\kappa$ : difusividade térmica do sistema; e

r: raio do poço.

A função $\mathrm{E}_{i}(-\mathrm{x})$ é uma integral do tipo

$$
\mathrm{E}_{i}(-x)=-\int_{x}^{\infty} \frac{e^{-u}}{u} d u
$$

que pode ser avaliada por métodos numéricos (Cavalcante, 2004).

$\mathrm{Da}$ Eq. (1), vemos que o termo $Q /(4 \pi K)$ é 0 coeficiente angular da reta obtida do gráfico de $\mathrm{T}\left(\mathrm{t}_{d}\right)$ versus $\mathrm{E}=\left[\mathrm{E}_{i}\left(-\mathrm{x}_{2}\right)-\right.$ $\mathrm{E}_{i}\left(-\mathrm{x}_{1}\right)$ ]. Comumente não se conhecem previamente os valores de $Q$ e K , de modo que, neste caso, para corrigir temperaturas de fundo de poço usando a Eq.(1), é necessário ter-se pelo menos duas medidas de temperatura oriundas duma mesma profundidade do poço, mas registradas em momentos distintos. A TEF $\left(T_{\infty}\right)$ é determinada pela interseção da reta ajustada com 0 eixo $\mathrm{E}=0,0$ que corresponde a um tempo de descanso infinito.

No conhecido método de Horner, faz-se uma aproximação assintótica da Eq.(1) obtendo-se a equação

$$
T\left(t_{d}\right)=T_{\infty}+\frac{Q}{4} \pi K \ln \frac{t_{d}+t_{c}}{t_{d}} \quad \text { para } t_{d}>0
$$

A condição para a Eq.(3) ser válida é que na Eq.(1)

$$
r^{2} /\left(4 \kappa t_{d}\right) \quad<<1 .
$$

Também neste método, Q e K não são previamente conhecidos, de modo que precisa-se de pelo menos duas medidas de $\mathrm{T}\left(\mathrm{t}_{d}\right)$ numa mesma profundidade para obter-se $\mathrm{T}_{\infty}$ por extrapolaçãa linear. Carvalho et al. (1980), Vacquier (1984), Carvalho et al. (1987), Deming \& Chapmann (1988a, b) e Lee et al. (1996), dentre outros, usaram o método de Horner para corrigir temperaturas de fundo de poço na determinação de fluxo de caIor terrestre. Este método foi também usado por Lachenbruch \& Brewer (1959), Lachenbruch \& Sass (1988) e Sass et al. (1988) para estimar a temperatura de equilíbrio a partir de perfis de temperatura da formação medida pelo método DST (sigla em inglês de drill stem test).

0 método de Horner (Eq.(3)), devido às simplificações envolvidas e, também, às considerações concernentes à geometria do fundo do poço e à taxa na qual o calor é extraído, não deve ser aplicado indiscriminadamente. Segundo Luheshi (1983), poços com $216 \mathrm{~mm}$ de diâmetro requerem tempos de descanso entre 20 e 50 h para que a Eq.(3) torne-se uma boa aproximação da Eq.(1).
A condição da Eq.(4) depende do quadrado do raio do poço, o que requer tempos de descanso mais longos para poços com maiores diâmetros. Tal exigência é contrária à prática de perfuração comumente utilizada, pois nela, à medida que aumenta a profundidade do poço seu diâmetro é reduzido progressivamente; e são exatamente nos diâmetros maiores que se utilizam tempos menores de descanso (Funnell et al., 1996). Segundo Roux et al. (1982), se $\mathrm{t}_{c} / \mathrm{t}_{d}<1 / 3$ para todos os pontos, a técnica de Horner dá resultados aceitáveis. Já Dowdle \& Cobb (1975), a partir de uma análise rigorosa da matemática governando o equilíbrio de TFP, mostraram que para $\left(t_{c} / t_{d}\right)>1 / 3$ - caso de muitos dos dados analisados neste trabalho - as extrapolações lineares com os dados do método de Horner tendem inerentemente à subestimar os vaIores da TEF. E a magnitude dessa tendência cresce quando $t_{c} / t_{d}$ cresce. Lachenbruch \& Brewer (1959), Shen \& Beck (1986), entre outros, acenaram que a precisão do método de Horner aumenta com 0 decréscimo da razão $t_{c} / t_{d}$ e Deming \& Chapman (1988a, b) mostraram que, na prática, medidas com tempos $t_{d}$ menores que 4 ou 5 horas são inadequadas para correções pelo método de Horner.

\section{A solução pelas integrais exponenciais}

A solução pelas integrais exponenciais da equação de Bullard desenvolvida neste trabalho visa reduzir, ou mesmo eliminar, algumas das restrições impostas pelo método de Horner. A solução para a Eq.(1) é obtida resolvendo-se numericamente as integrais exponenciais $\mathrm{E}_{i}(-\mathrm{x})$ pelo método trapezoidal (Cavalcante, 2004). A vantagem em usar-se a Eq.(1) é que assim não há restrições para 0 valor de $t_{d}$ podendo aproveitar-se dados de temperatura de fundo de poço com tempos de descanso relativamente pequenos quando comparados ao tempo necessário para 0 equilíbrio térmico do poço.

A acurácia do método depende da confiança e da acurácia das medidas de $\mathrm{T}, \mathrm{t}_{c}$ e $\mathrm{t}_{d}$ que são realizadas e informadas pelas empresas de perfuração. Quando três ou mais pontos são disponíveis para uma mesma profundidade pode-se calcular um desvio padrão para a TEF baseado na dispersão dos pontos em torno da linha de melhor ajuste.

\section{Dados experimentais}

Um total de 577 registros sobre temperatura de fundo de poço oriundos de 207 poços (continentais e costeiros) de diversos campos de petróleo das bacias sedimentares do Recôncavo, Camamu, Almada, Cumuruxatiba e Jequitinhonha foi utilizado neste trabalho. Esses registros, para cada poço, consistem em medidas 
da temperatura e respectiva profundidade, raio do poço, tempo de circulação da lama e tempo de descanso do poço. Os dados de temperatura foram obtidos de poços de profundidades entre $50 \mathrm{e}$ $5.000 \mathrm{~m}$, sendo a maior parte deles provenientes de profundidades entre 1.000 e $3.000 \mathrm{~m}$.

Uma análise criteriosa prévia dos dados levou à rejeição de uma quantidade deles por apresentarem valores questionáveis. São exemplos, os registros incoerentes de tempo, $\mathrm{t}_{c}$ ou $\mathrm{t}_{d}-0$ fato de o poço estar no estado transiente de propagação do calor, qualquer erro no registro do tempo implica numa determinação inadequada da temperatura da formação. Outro tipo de erro observado é ter-se um mesmo valor para a temperatura em diferentes corridas (tempos) dos perfis. Dados podem ser fabricados como, por exemplo, três diferentes perfis mostrando temperaturas do tipo $80,90,100^{\circ} \mathrm{C}$.

Do total de 577 registros, 412 apresentam pelo menos dois registros de temperatura para uma mesma profundidade, medidas em instantes diferentes depois de cessada a circulação da lama. Destes 412 dados, 196 consistem em 98 pares de temperatura, 129 continham 43 grupos de três temperaturas, 52 dados tinham 13 grupos de quatro temperaturas e 35 apresentavam 7 grupos de cinco temperaturas. Como cada amostra de múltiplas temperaturas possibilita a determinação de uma única temperatura estática, os 412 dados permitiram determinar 161 temperaturas estáticas para diferentes níveis de profundidades dos diferentes poços.

Dos outros 165 registros, um grupo contem os valores de r, $\mathrm{t}_{c}$ e $\mathrm{t}_{d}$ mas apenas uma única medida de temperatura proveniente de uma profundidade qualquer e num outro grupo falta o registro do tempo de circulação da lama.

\section{Tratamento dos dados}

A variabilidade da qualidade dos dados de temperatura de fundo de poço levou-nos a utilizar metodologias que se adequassem ao tipo de dados em questão.

Para os dados de temperatura que continham informações completas, ou seja, pelo menos dois registros de temperaturas oriundos de uma mesma profundidade do poço, o raio do poço, os tempos de circulação da lama e 0 de descanso do poço, fizemos a correção resolvendo a Eq.(1) por métodos numéricos (Cavalcante, 2004). No uso desta equação fizemos uma aproximação quanto ao valor da difusividade térmica $(\kappa)$. Esta difusividade para rochas in situ varia muito pouco, tipicamente em torno de $1,1 \times 10^{-6} \mathrm{~m}^{2} \mathrm{~s}^{-1}$ (Kappelmeyer e Haenel, 1974) e na água

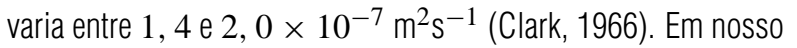
trabalho, usamos um valor médio de $5 \times 10^{-7} \mathrm{~m}^{2} \mathrm{~s}^{-1}$ para o sis- tema composto pela lama e formação, como sugerido por Luheshi (1983). A TEF foi determinada através da reta melhor ajustada pelos mínimos quadrados. A Figura 1 ilustra graficamente a aplicação do método de correção baseado nas integrais exponenciais em dados de TFP provenientes de diferentes profundidades de alguns poços selecionados. Cada ponto indica uma TFP registrada num determinado instante numa profundidade particular, que é indicada ao lado das medidas. Cada reta corresponde àquela que melhor se ajusta pelo método dos mínimos quadrados aos dados de TFP oriundos de uma mesma profundidade do poço. A interseção de cada reta com o eixo das ordenadas fornece a temperatura corrigida (supostamente a de equilíbrio).

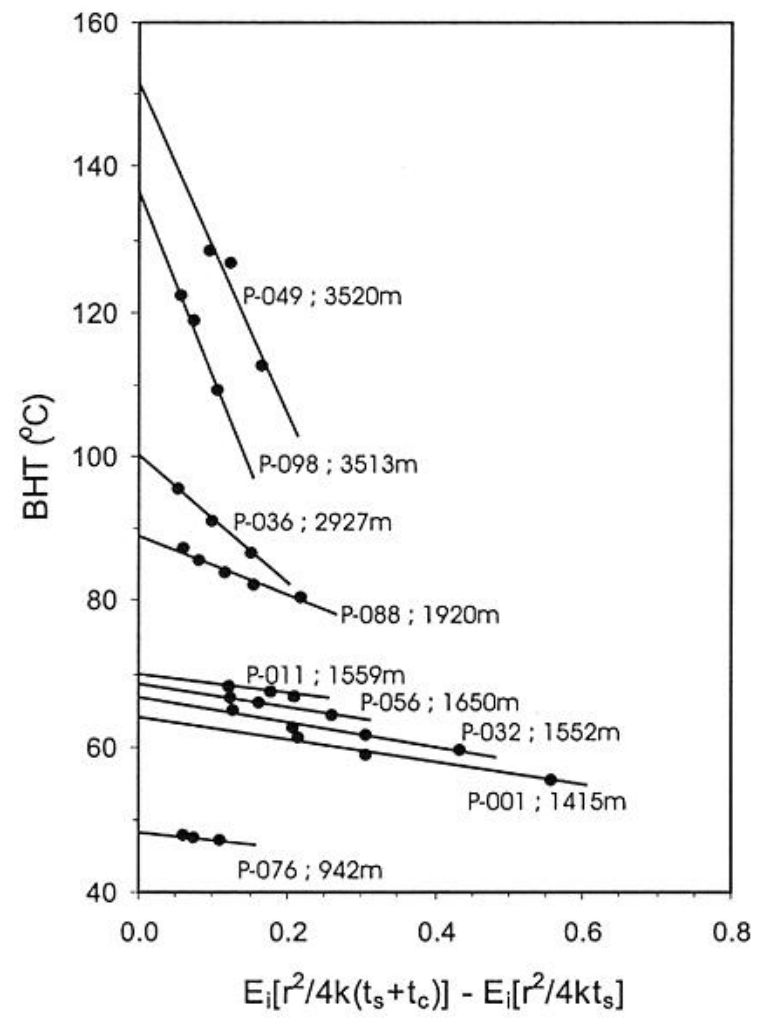

Figura 1 - Gráficos exemplificando a correção pelas integrais exponenciais para poços selecionados. Cada ponto nos gráficos indica uma TFP registrada numa profundidade particular do poço, que é indicada ao lado do nome do poço. A abscissa é o termo $E_{i}\left\{-r^{2} /\left[4 \kappa\left(t_{d}+t_{c}\right)\right\}-E_{i}\left[-r^{2} /\left(4 \kappa t_{d}\right)\right] d a\right.$ Eq.(1).

Figure 1 - Plots exemplifying the exponential integral correction for selected wells. Each point on the plots indicates a BHT registered in a particular depth of the well, which is indicated at the side of the name of the well. The abscissa is the term $E_{i}\left\{-r^{2} /\left[4 \kappa\left(t_{d}+t_{c}\right)\right\}-E_{i}\left[-r^{2} /\left(4 \kappa t_{d}\right)\right]\right.$ of Eq.(1).

A Figura 2 mostra os gráficos dos dados de temperatura de fundo de poço não-corrigidos - tomando-se as maiores temperaturas das séries de múltiplas temperaturas, presumivelmente as mais próximas da TEF - e destas mesmas temperaturas corrigidas 


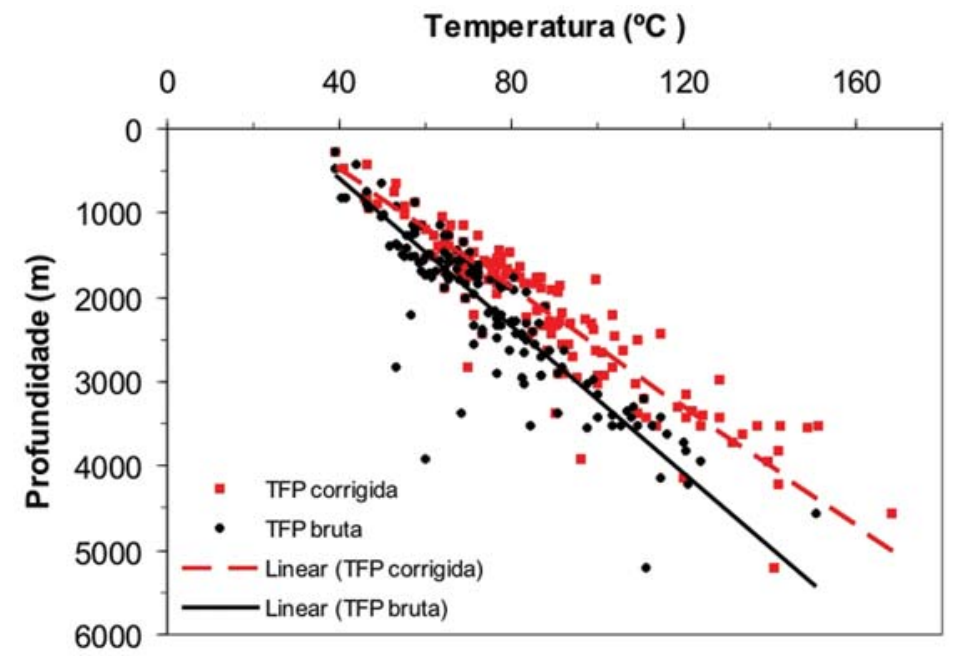

Figura 2 - Temperaturas de fundo de poço corrigidas pela solução das integrais exponenciais $(\boldsymbol{\square})$ e não-corrigidas $(\mathbf{O})$ das cinco bacias estudadas. Um ajuste pelos mínimos quadrados produziu um gradiente geotérmico de $(28,3 \pm 1,6) \times 10^{-3}{ }^{\circ} \mathrm{C}$ $\mathrm{m}^{-1}, \mathrm{R}^{2}=0,84$, para as temperaturas corrigidas (linha interrompida) e de $(22,9 \pm 1,5) \times 10^{-3}{ }^{\circ} \mathrm{C} \mathrm{m}-1, \mathrm{R}^{2}=0,74$, para as não-corrigidas (linha cheia).

Figure 2 - Bottom hole temperatures corrected by the exponential integral solution method ( $)$ and not-corrected (-) from the five basins studied. A least squares fit yields a geothermal gradient of $(28,3 \pm 1,6) \times 10^{-3}{ }^{\circ} \mathrm{C} \mathrm{m}-1, R^{2}=0,84$, to the corrected temperatures (dotted line) and of $(22,9 \pm 1,5) \times 10^{-3}{ }^{\circ} \mathrm{Cm}^{-1}, R^{2}=0,74$, to the not-corrected ones (full line).

pelo método das integrais exponenciais, ambos versus a profundidade do poço.

Para os dados de temperatura que continham os valores de $r, t_{c}$ e $t_{d}$ mas apenas uma única medida da temperatura proveniente de uma profundidade qualquer, utilizamos o esquema de correção tempo-profundidade, semelhante ao proposto por Deming \& Chapman (1988b). Este método consiste em obter-se uma correlação entre os coeficientes angulares $(Q / 4 \pi K)$ obtidos da Eq.(1) usando-se os dados disponíveis de múltiplas leituras de temperaturas e as profundidades correspondentes.

A idéia por trás do esquema da correção tempo-profundidade é obter uma equação empírica que nos dê um coeficiente angular médio para a Eq.(1) como uma função da profundidade e usar este coeficiente para correções na área estudada quando dispusermos de apenas um registro de temperatura. A equação desejada deve ser forçada a produzir um coeficiente angular nulo para a profundidade zero, já que a temperatura da lama de perfuração no fundo de um poço extremamente raso deve ser aproximadamente igual à temperatura da superfície. Então, o coeficiente angular precisa crescer com a profundidade, pois a lama fria de perfuração interage progressivamente com temperaturas mais altas nas maiores profundidades.

Para construir a correlação, nós separamos os dados dos poços em três grupos, de acordo com o diâmetro do poço: poços com diâmetros de 21,11 cm, de $31,11 \mathrm{~cm}$ e todos os poços reunidos sem distinção do diâmetro. A Figura 3 apresenta as curvas correspondentes a um ajuste polinomial de segundo grau para os dados dos três grupos de poços. As curvas da Fig. 3 foram ajustadas pela função quadrática

$$
\mathrm{A}=\alpha z+\beta z^{2}
$$

onde A é o coeficiente angular $Q / 4 \pi \mathrm{K}$ da Eq.(1) e z a profundidade; para $A$ em graus Celsius, e z em metros, a regressão pelos mínimos quadrados sobre os pontos dos três grupos mostrados na Figura 3 produziram para os coeficientes $\alpha$ e $\beta$ os valores mostrados na Tabela 1.

Tabela 1 - Coeficientes $\alpha$ e $\beta$ ajustados para a equação $A=\alpha z+\beta z^{2}$ no esquema tempo-profundidade da Figura 2 para poços com diâmetro de 21,11 cm, 31,11 cm e para todos os diâmetros juntos.

Table 1 - Fitted coefficients $\alpha$ and $\beta$ to the equation $A=\alpha z+\beta z^{2}$ in the time-depth scheme of Figure 2 for well diameters of $21,11 \mathrm{~cm}, 31,11 \mathrm{~cm}$ and all diameters together.

\begin{tabular}{|c|c|c|}
\hline Diâmetro $(\mathrm{cm})$ & $\alpha\left({ }^{\circ} \mathrm{C} \mathrm{m}^{-1}\right)$ & $\beta\left({ }^{\circ} \mathrm{C} \mathrm{m}^{-2}\right)$ \\
\hline 21,11 & $-2,03 \times 10^{-6}$ & 0,0258 \\
\hline 31,11 & $-1,14 \times 10^{-6}$ & 0,0338 \\
\hline Todos os diâmetros & $-3,96 \times 10^{-6}$ & 0,0345 \\
\hline
\end{tabular}




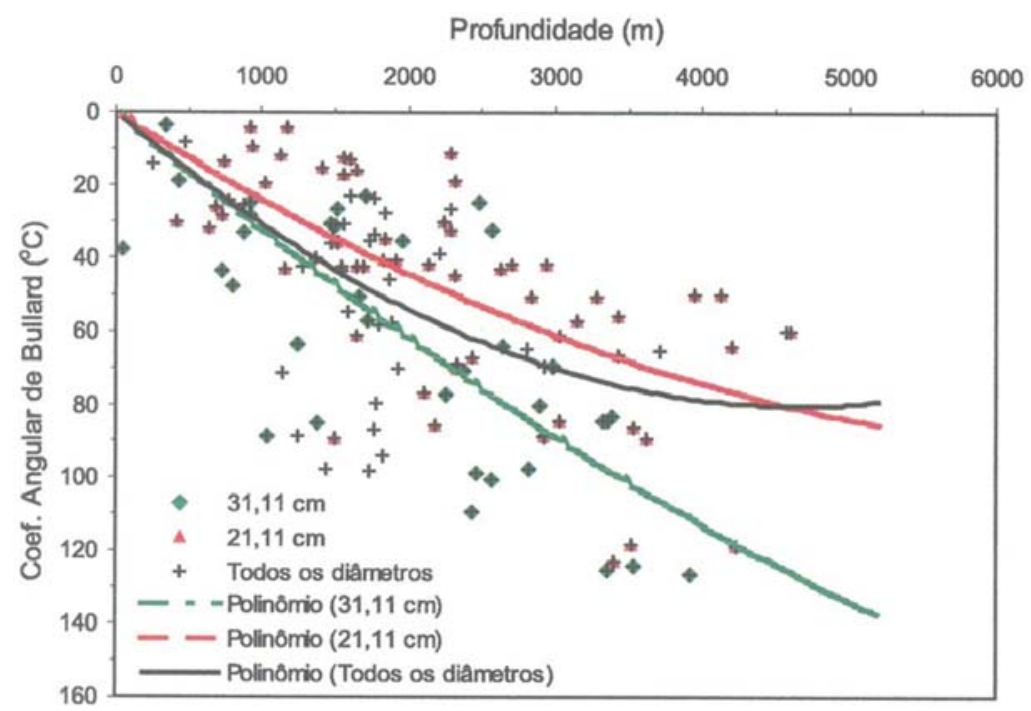

Figura 3 - Coeficiente angular da equação de Bullard (1947) como função da profundidade para poços com diâmetro de 21,11 cm ( $\mathbf{\Lambda}), 31,11 \mathrm{~cm}(\boldsymbol{\bullet})$ e para todos os diâmetros juntos (十) das cinco bacias estudadas. As curvas foram ajustadas pelos mínimos quadrados.

Figure 3 - Angular coefficients of Bullard (1947) equation as function of depth for well diameters of $21,11(\mathbf{\Delta}) \mathrm{cm}, 31,11 \mathrm{~cm}$ -) and all diameters together $(+)$ from the five studied basins. Curves were least squares fitted.

Os pontos na Figura 3 mostram um grande espalhamento que deve resultar de várias fontes. Parte da dispersão observada pode ser conseqüência da variação (em tempo e localização) das propriedades térmicas da formação (por exemplo, causada por fluxo de fluidos durante equilíbrio) e da lama de perfuração. Segundo Deming (1989), tentativas de explicar esse grande espalhamento pelo número de pontos com que é determinado o coeficiente angular, ou pelo raio do poço são infrutíferas. A correlação com a profundidade é fraca, mas ela existe.

A Figura 4 mostra os gráficos das temperaturas de fundo de poço com um único registro por profundidade corrigidas pelo esquema de tempo-profundidade e dessas temperaturas nãocorrigidas ambos em função da profundidade.

Para um conjunto de dados, faltam os tempos de circulação da lama. Na falta deste tempo vários pesquisadores contornaram esta dificuldade usando um tempo de circulação padrão para todas as correções. Chapman etal. (1984) e Reiter \& Jessop (1985) usaram 4 horas; Deming \& Chapman (1988a,b), Deming (1989) e Lee et al., (1996) usaram 5 horas. Hermanrud et al. (1990) propuseram a fórmula empírica

$$
t_{c}=(1,3+D) /(1,3-0,091 \times D)
$$

para estimar o valor de $t_{c}$, onde $D$ é a profundidade indicada pelo cabo de perfuração em quilômetros e $t_{c}$ é dado em horas. É evidente nesta expressão uma significante incerteza associada ao va-
Ior de $t_{c}$. Uma aparente justificativa para essas práticas é que a temperatura de equilíbrio determinada pela equação de Bullard varia pouco quando 0 tempo de circulação varia. Isto não é 0 mesmo que dizer que a acurácia do método de correção não é afetada quando um tempo de circulação incorreto é usado. Nós optamos por obter um valor de $t_{c}$ característico com base nos valores conhecidos deste tempo em nossa área de estudo. No histograma da Figura 5, construído com os tempos de circulação da lama que nos foram disponibilizados neste estudo, os valores de $\mathrm{t}_{c}$ no histograma variam de 0,5 a $10 \mathrm{~h}$ mas há um nítido valor característico em torno de $2 \mathrm{~h}$. Então, usamos este valor de $2 \mathrm{~h}$ para 0 tempo de circulação onde este parâmetro não foi informado.

\section{RESULTADOS E DISCUSSÃO}

A comparação das temperaturas brutas de fundo de poço com as temperaturas estáticas da formação obtidas pela aplicação de correção mostra que aquelas são sistematicamente menores que as temperaturas corrigidas (Fig. 6). Como enfatizaram Hermanrud etal. (1990), essas diferenças entre os valores das temperaturas de fundo de poço e das temperaturas estáticas são suficientemente grandes para alterar o prognóstico de geração de petróleo quando baseado em modelagem da história térmica da bacia calibrada por dados de temperatura. Para profundidades até $1 \mathrm{~km}$, vê-se que as correções ficam entre zero e $5^{\circ} \mathrm{C}$; já para profundi- 


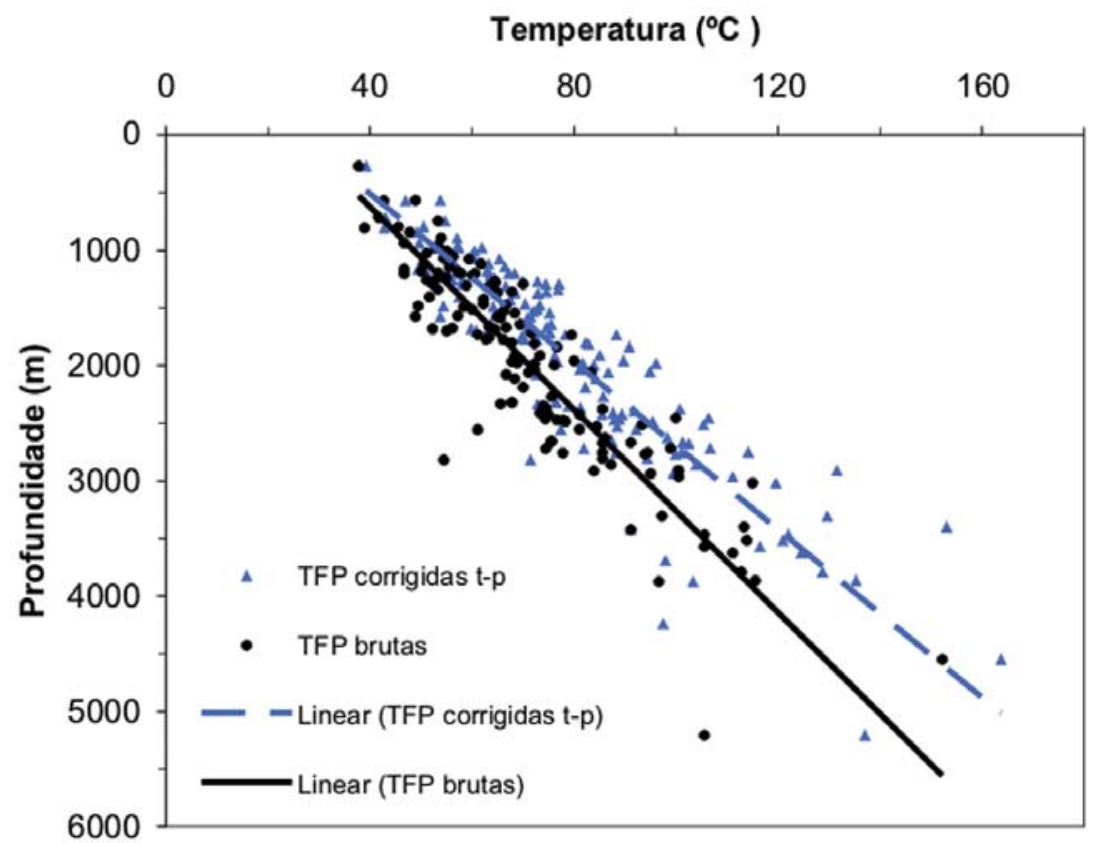

Figura 4 - Gráficos das TFPs com um único registro por profundidade corrigidas pelo esquema de tempo-profundidade ( $\mathbf{\Delta}$ ) e das temperaturas não-corrigidas $(\bullet)$, ambos em função da profundidade. Um ajuste pelos mínimos quadrados produziu um gradiente geotérmico de $(27,5 \pm 1,4) \times 10^{-3}{ }^{\circ} \mathrm{C} \mathrm{m}^{-1}, \mathrm{R}^{2}=0,80$, para as temperaturas corrigidas (linha interrompida) e de $(22,7 \pm 1,3) \times 10^{-3}{ }^{\circ} \mathrm{C} \mathrm{m}{ }^{-1}, R^{2}=0,74$, para as não-corrigidas (linha cheia).

Figure 4 - Plots of BHTS with a single register for depth corrected by the time-depth scheme ( $\mathbf{\Delta}$ ) and non-corrected temperatures -), both against depth. A least squares fit yields a geothermal gradient of $(27,5 \pm 1,4) \times 10^{-3}{ }^{\circ} \mathrm{Cm}^{-1}, R^{2}=0,80$, to the corrected temperatures (dotted line) and of $(22,7 \pm 1,3) \times 10^{-3}{ }^{\circ} \mathrm{C} \mathrm{m}^{-1}, R^{2}=0,74$, to the non-corrected ones (full line).

dades maiores as correções são praticamente independentes da profundidade, variando de menos de $1^{\circ} \mathrm{C}$ e chegando a valores próximos a $25^{\circ} \mathrm{C}$, com maior concentração na faixa até $17^{\circ} \mathrm{C}$, como pode ser constatado na Figura 6 .

0 erro presente nos valores da temperatura $T_{\infty}$ após a aplicação da correção, é de quase impossível estimativa, haja vista as incertezas na medida da TFP e nas medidas dos vários parâmetros envolvidos no cálculo das correções, às quais se somam às incertezas sistemáticas dos procedimentos de correção. Deming (1989), que corrigiu dados de TFP usando o método aproximado de Horner e tempo de circulação padrão de $5 \mathrm{~h}$, salienta que, apesar de um estudo definitivo não ter sido feito, o erro médio das temperaturas corrigidas é provavelmente da ordem de $\pm 5^{\circ} \mathrm{C}$, dependendo das condições locais e do tipo de procedimento de correção utilizado. Este erro médio leva a um desvio relativo melhor que $10 \%$ para as temperaturas na profundidade de $500 \mathrm{~m}$ e melhor que $5 \%$ além de $2.500 \mathrm{~m}$. Em nosso trabaIho, onde a correção foi feita pela solução da Eq.(1) e dispusemos do tempo de circulação da lama para a maioria dos poços, 0 erro médio poderia ser melhor que $\pm 5^{\circ} \mathrm{C}$, talvez $2 \mathrm{a} 3^{\circ} \mathrm{C}$.
Os métodos de correção, teóricos ou empíricos, partem do princípio que uma TFP registrada nas condições de perfuração é inferior à temperatura verdadeira da formação - 0 que é correto - e as fórmulas desenvolvidas para corrigir esta temperatura atentando para as medidas que são disponíveis no processo de perfuração. A acurácia da medida resultante teria de ser avaliada por comparação do valor corrigido com 0 valor verdadeiro da formação - após feita a correção, este valor verdadeiro pode ser determinado, por exemplo, fazendo-se uma nova medida da temperatura num dado ponto após um tempo de descanso superior ao tempo necessário para estabilização térmica da formação - ou usando-se métodos como o DST (sigla em inglês de drill stem tesh), considerado dar a medida mais acurada da formação em tempos curtos após a perfuração. Não temos referência se esta comparação já foi feita.

Na Figura 2, as retas ajustadas pelos mínimos quadrados dão para as temperaturas corrigidas pela solução das integrais exponenciais um gradiente de $(28,3 \pm 1,6) \times 10^{-3}{ }^{\circ} \mathrm{C} \mathrm{m}^{-1}$ e para as temperaturas não-corrigidas um gradiente de $(22,9 \pm 1,5) \times$ $10^{-3}{ }^{\circ} \mathrm{C} \mathrm{m}^{-1}$. Comparando os dois gradientes, 0 de maior valor 


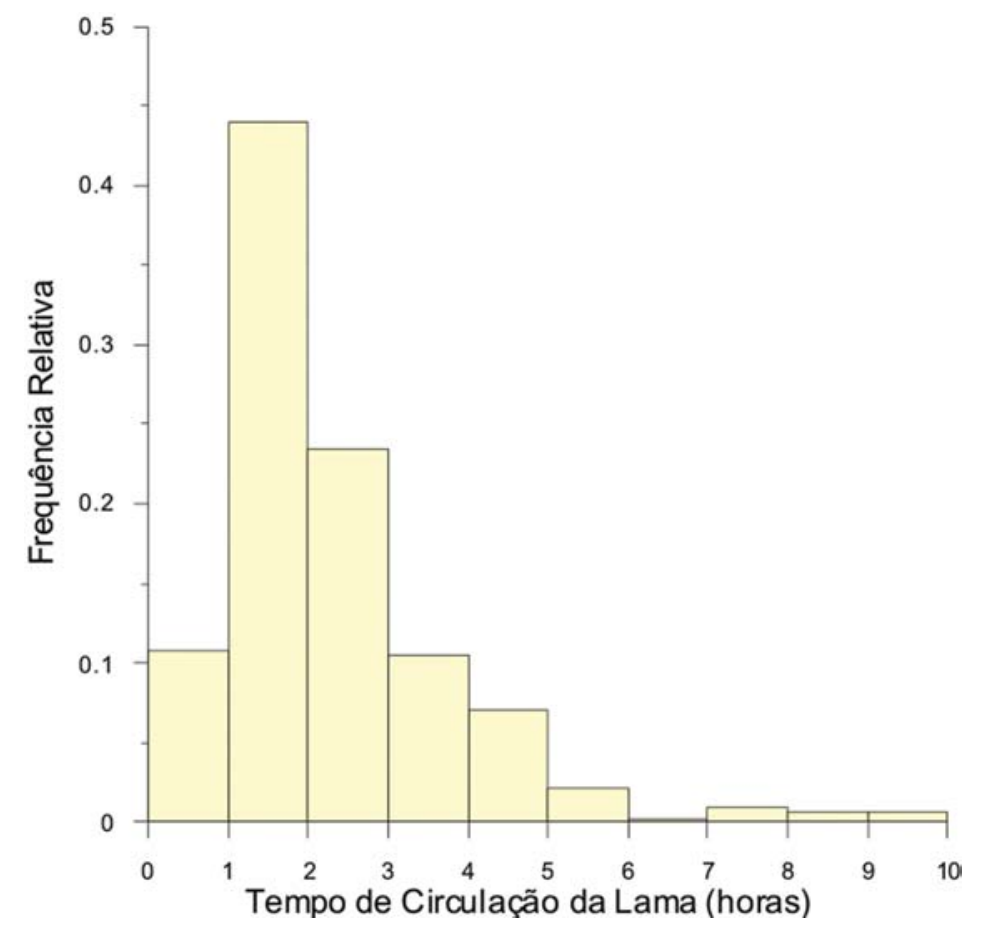

Figura 5 - Histograma dos dados de tempo de circulação da lama para todos os poços das cinco bacias. Figure $\mathbf{5}$ - Circulation time data including all wells from the five basins.

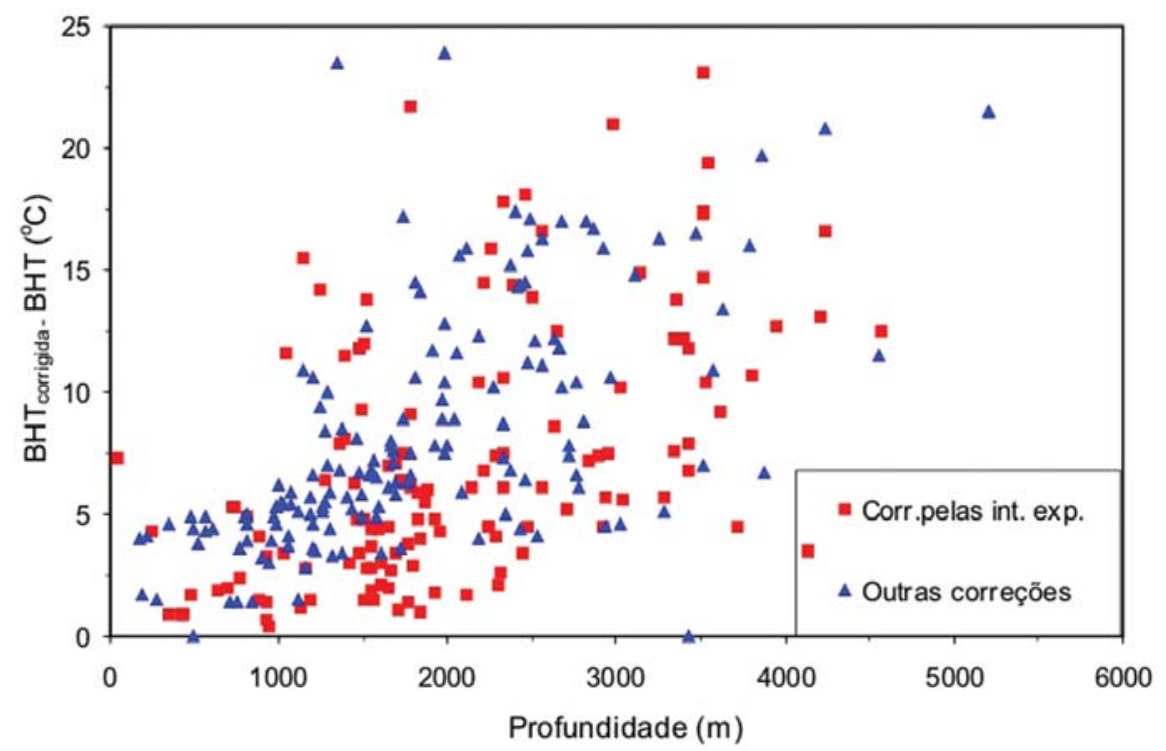

Figura 6 - Gráfico das correções das TFP feitas pelo método das integrais exponenciais ( $\mathbf{\square})$ e pelo esquema tempo-profundidade (ム) ( $T_{\text {corr }}-T_{\text {não-corr }}$ ), versus a profundidade. No primeiro caso, tomou-se para subtração a temperatura mais alta entre as múltiplas.

Figure $\mathbf{6}$ - Plot of the BHT corrections made by the exponential integral solution method (-) and by the time-depth scheme ( $\mathbf{\Delta})$, $\left(T_{\text {corr }}-T_{\text {non-corr }}\right)$, versus depth. In the first case, we consider the higher temperature between the multiple ones to subtraction. 
é 0 correspondente às temperaturas corrigidas, como seria esperado; suas faixas de valores discrepam em mais de dois desvios padrões, o que demonstra a importância de corrigir-se as TFPs.

$\mathrm{Na}$ Figura 4, para as temperaturas corrigidas pelo esquema de tempo-profundidade o gradiente geotérmico médio obtido por regressão linear é de $(27,5 \pm 1,4) \times 10^{-3}{ }^{\circ} \mathrm{C} \mathrm{m}^{-1}$, 0 qual coincide em menos de um desvio padrão com 0 de $(28,3 \pm$ $1,6) \times 10^{-3}{ }^{\circ} \mathrm{C} \mathrm{m}^{-1}$ obtido com a correção pela solução das integrais exponenciais vista na Fig. 2. Para as TFP brutas, 0 gradiente geotérmico médio é de $(22,7 \pm 1,3) \times 10^{-3}{ }^{\circ} \mathrm{C} \mathrm{m}^{-1}$, valor praticamente igual ao correspondente às temperaturas brutas na Fig. 2.

Tomando-se os gradientes geotérmicos médios obtidos com as temperaturas corrigidas nas Figs. 2 e 4, obtivemos para 0 gradiente geotérmico médio em toda a área estudada 0 valor de $(27,9 \pm 2,1) \times 10^{-3}{ }^{\circ} \mathrm{C} \mathrm{m} \mathrm{m}^{-1}$. Este valor está estatisticamente dentro da medida de $(25,1 \pm 5,6) \times 10^{-3}{ }^{\circ} \mathrm{C}$ $\mathrm{m}^{-1}$ calculada com dados da Petrobrás (dados não publicados) para 0 gradiente geotérmico médio da bacia de Cumuruxatiba. Ele é também coincidente com o gradiente geotérmico médio de $(28,0 \pm 2,4) \times 10^{-3}{ }^{\circ} \mathrm{C} \mathrm{m}^{-1}$ obtido por Fontes (1980) para a bacia sedimentar Sergipe-Alagoas.

Para verificar a eficácia do esquema tempo-profundidade, corrigimos com ele os dados com múltiplas TFP numa mesma profundidade e comparamos o gradiente médio obtido com este esquema com aquele obtido para as temperaturas corrigidas na Fig. 2. Para isso, em cada série, utilizamos a temperatura de maior valor entre as múltiplas temperaturas numa profundidade - é a temperatura registrada com um maior tempo de descanso e que, presumivelmente, está mais próxima da temperatura de equilíbrio. Conhecendo a profundidade em que foi feita a medida, calculamos 0 coeficiente angular com a Eq.(4) usando, para isso, os vaIores $\alpha$ e $\beta$ da Tab. 1 e tomamos este coeficiente como igual a $Q / 4 \pi \mathrm{K}$ na Eq.(1) para calcular $\mathrm{T}_{\infty}$.

A Figura 7 mostra o gráfico das maiores temperaturas de cada série de múltiplas temperaturas corrigidas pelo método de tempoprofundidade versus a profundidade. 0 gradiente geotérmico médio, obtido por regressão linear, é de $(29,0 \pm 1,7) \times$ $10^{-3}{ }^{\circ} \mathrm{C} \mathrm{m}^{-1}, 0$ qual apresenta uma boa concordância com 0 gradiente de $(28,2 \pm 1,6) \times 10^{-3}{ }^{\circ} \mathrm{C} \mathrm{m}^{-1}$ obtido pela solução das integrais exponenciais. Este resultado respalda 0 uso deste método como feito neste trabalho.

Analisando as correções de temperatura feitas pelo dois métodos, verifica-se que aquelas feitas pelo esquema de tempoprofundidade apresentam maiores magnitudes na estimativa das temperaturas estáticas, com um acréscimo médio de 13,3\%, en- quanto que nas feitas pela solução das integrais exponenciais a correção média é de 8,3\%. Por outro lado, a correção média para poços com diâmetro de $31 \mathrm{~cm}$ foi de 13,7\%, enquanto que para poços de $21 \mathrm{~cm}$ de diâmetro a correção foi de 10\%, um resultado já esperado.

\section{CONCLUSÕES}

Neste trabalho, investigamos um banco de dados de 577 registros de temperatura de fundo de poço provenientes das bacia do Recôncavo, Camamu, Almada, Cumuruxatiba e Jequitinhonha, buscando determinar as temperaturas estáticas das formações, TEF. No total, 307 valores de TEF foram determinados os quais poderão ser usados em estudos geotérmicos da região.

As correções realizadas ficam entre zero e $5^{\circ} \mathrm{C}$ para profundidades até $1 \mathrm{~km}$; já para profundidades maiores as correções são praticamente independentes da profundidade, variando de menos de $1^{\circ} \mathrm{C}$ e chegando a valores próximos a $25^{\circ} \mathrm{C}$, com maior concentração na faixa até $17^{\circ} \mathrm{C}$.

As medidas dos gradientes geotérmicos obtidos com as temperaturas corrigidas pelo método das integrais exponenciais e pelo esquema de tempo-profundidade foram, respectivamente, $(28,3 \pm 1,6) \times 10^{-3}{ }^{o} \mathrm{C} \mathrm{m}^{-1}$ e $(27,5 \pm 1,4) \times 10^{-3}{ }^{\circ} \mathrm{C} \mathrm{m}^{-1}$, com uma discrepância relativa de 2,8 \% entre os valores médios dos gradientes. A discrepância relativa desses valores médios com os dos gradientes para as temperaturas não corrigidas é em torno de $18 \%$, um valor típico para essas correções.

Esses gradientes são estatisticamente iguais, o que comprova a validade do uso do esquema de tempo-profundidade. A vantagem de fazer-se a correção pelo esquema de tempo-profundidade é que ele utiliza equações calibradas a partir de dados da própria bacia em estudo e inclui, no processo de correção, os parâmetros profundidade, raio do poço e tempos de circulação e descanso do poço. Entretanto, não é adequado usar a equação calibrada para uma bacia em outras bacias, a não ser que elas possuam um contexto geológico-geofísico similar. Por exemplo, o gradiente de $(27,5 \pm 1,4) \times 10^{-3}{ }^{\circ} \mathrm{C} \mathrm{m}^{-1}$ obtido com este esquema neste estudo coincide estatisticamente com de $(28,0 \pm 2,4) \times 10^{-3}{ }^{\circ} \mathrm{C}$ $\mathrm{m}^{-1}$ obtido por Fontes (1980) para a bacia Sergipe-Alagoas.

A precisão das medidas das temperaturas corrigidas com os métodos utilizados neste estudo é de difícil avaliação devido às incertezas não conhecidas dos vários parâmetros envolvidos na correção. Quando se tem três ou mais temperaturas numa profundidade, é possível calcular o desvio padrão para a temperatura corrigida obtida na interseção feita por extrapolação com regressão linear. Fora disso, é impossível avaliar-se a precisão. 


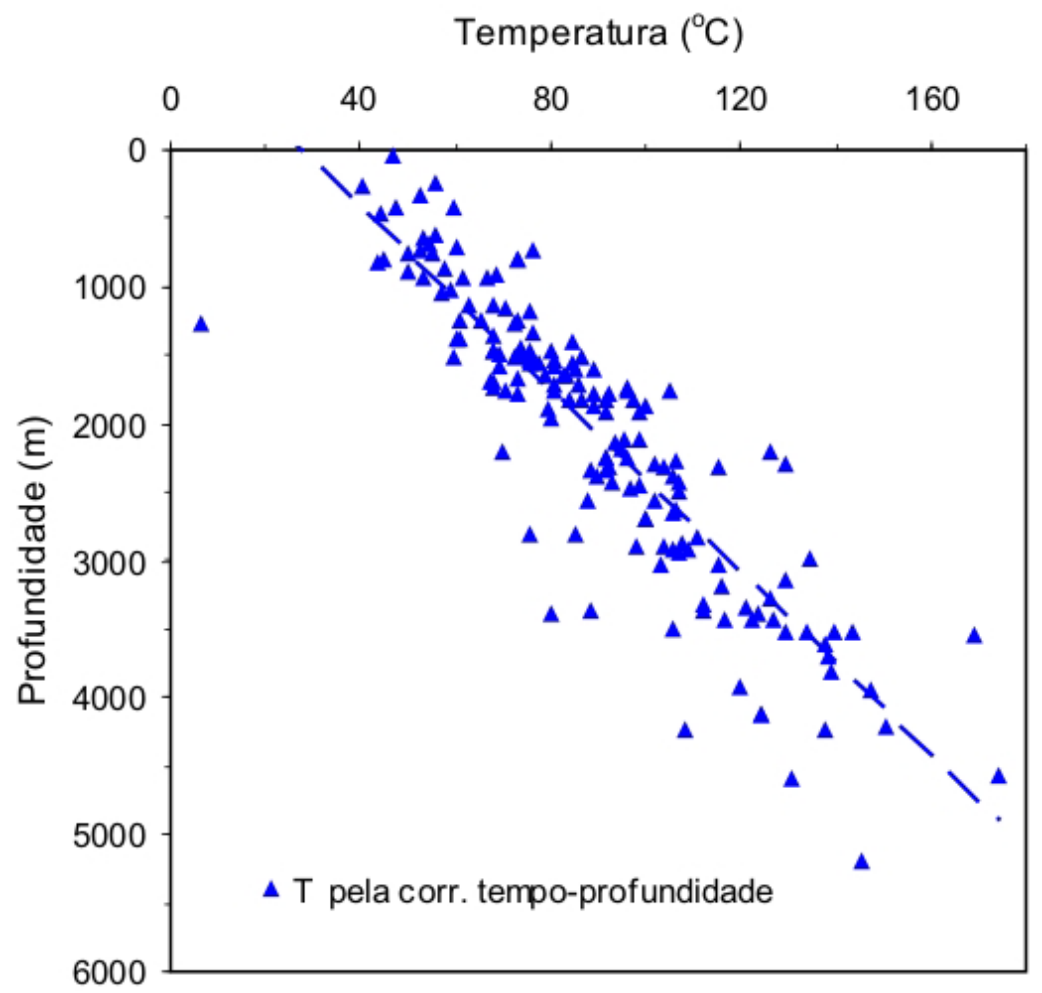

Figura 7 - Gráfico da aplicação do esquema de correção tempo-profundidade aos dados de múltiplas TFP numa mesma profundidade. Para a aplicação, tomou-se a temperatura mais alta. 0 ajuste pelos mínimos quadrados deu um gradiente de $(29,0 \pm 1,7) \times 10^{-3}{ }^{\circ} \mathrm{C} \mathrm{m}^{-1}$ com $^{2}=0,78$.

Figure 7 - Plot of the application of the time-depth correction scheme to multiples BHT in a same depth. For the application, one used the higher temperature. A least squares fit yields a gradient of $(29,0 \pm 1,7) \times 10^{-3}{ }^{\circ} \mathrm{Cm}^{-1} \mathrm{com} R^{2}=0,78$.

Para avaliar a acurácia de nossos métodos precisaríamos comparar uma ou mais de nossas temperaturas corrigidas com as temperaturas verdadeiras da formação correspondente obtidas por algum outro método, DST, por exemplo. Infelizmente não dispusemos de tal informação.

\section{AGRADECIMENTOS}

Os autores agradecem à Petrobras pela disponibilização dos dados de TFP utilizados neste trabalho e a seu técnico Guilherme Gontijo pelo apoio na coleta desses dados. 0 senhor Anderson Gusmão realizou este trabalho como bolsista de graduação do PRH08 da ANP e o trabalho foi custeado com recursos do projeto GEOTERM envolvendo a Finep (convênio 21.01.0292.00) e a Petrobras (termo de compromisso 650.4.113.014).

\section{REFERÊNCIAS}

BULLARD EC. 1947. The time necessary for a borehole to attain temperature equilibrium. Mon. Not. Royal Astr. Soc., 5: 127-130.
CARVALHO HS, PURWOKO, SISWOYO, THAMRIN M \& VACQUIER V. 1980. Terrestrial heat flow in the tertiary Basin of Central Sumatra. Tectonophysics, 69: 163-188.

CARVALHO HS, LÔBO PFS, CAMPOS JNP \& ZEMBRUSCKI SF. 1987. Estudo do fluxo de calor e movimentação de flú́dos na bacia do Médio Amazonas. Revista Brasileira de Geofísica, 5: 231-243.

CAVALCANTE AG. 2004. Correção de dados de temperatura de fundo de poço (BHT). Trabalho de graduação, curso de graduação em geofísica, UFBa, $75 \mathrm{pp}$.

CHAPMAN DS, KEHO TH, BAUER MS \& PICARD MD. 1984. Heat flow in the Uinta Basin determined from bottom hole temperature (BHT) data. Geophysics, 49: 453-466.

CLARK SP Jr. 1966. Thermal conductivity, em Handbook of Physical Constants, ed. Por S.P. Clark Jr., Geol. Soc. of Am. Mem. 97: 459-482.

DEMING D \& CHAPMAN DS. 1988a. Inversion of bottom-hole temperature data - the Pineview field, Utha-Wyoming thrust belt. Geophysics, 53(5): 707-720. 
DEMING D \& CHAPMAN DS. 1988b. Heat flow in the Utah-Wyoming thrust belt from analysis of bottom-hole temperature data measured in oil and gas wells. J. Geophys. Res., 93 (B11): 13657-13672.

DEMING D. 1989. Application of bottom-hole temperature corections in geothermal studies. Geothermics, 18: 775-786.

DOWDLE WL \& COBB WM. 1975. Static formation temperature from well logs-an empirical method. J. of Petroleum Technology, 27(11): 13261330.

FONTES LCAA. 1980. Determinação do fluxo geotérmico na bacia sedimentar Sergipe-Alagoas. Dissertação de mestrado, UFBa.

FUNNELL R, CHAPMAN D, ALLIS R \& ARMSTRONG P. 1996. Thermal state of the Taranaki Basin, New Zealand. J.Geophys. Res., 101(B11): 25.197-25.215

HERMANRUD C, CAO S \& LERCHE I. 1990. Estimates of virgin rock temperature derived from BHT measurements: bias and error. Geophysics, 55: 924-931.

KAPPELMEYER 0 \& HAENEL R. 1974. Geothermics with special reference to applications. Gebrüder Borntraeger, Berlin, Geoexploration Monographs, Series 1, No. 4, 238 pp.

LACHENBRUCH AH \& BREWER MC. 1959. Dissipation of the temperature effect of drilling a well in Aretia Alaska. U. S. Geol. Survey Bull., 1083-C: 73-109.

LACHENBRUCH AH \& SASS JH. 1988. The stress heat flow paradox and thermal results from Cajon Pass. Geophys. Res. Lett., August Supl., 15(9): 981-984.

LEE Y, DEMING D \& CHEN KF. 1996. Heat flow and heat production in the Arkoma Basin and Ohlahoma Plataform, sutheastern Oklahoma. J. Geophys. Res., 101: 25387-25401.

LUHESHI MN. 1983. Estimation of formation temperature from borehole measurements: Geophys. J. R the Astron. Soc., 74: 747-776.

RAYMOND LR. 1969. Temperature distribution in a circulating drilling fluid. Journal of Petroleum Technology, 21(3): 333-341.

REITER M \& JESSOP AM. 1985. Estimates of terrestrial heat flow in offshore eastern Canada. Can. J. Earth Sci., 22: 1503-1517.

ROUX B, SANYAL SK \& BROWN SL. 1982. An improved approach to estimating true reservoir temperature from transient temperature data. Em Geothermal Log Interpretation Handbook, ed. JU.K. Hallenburg, pp. V53-60.

SASS JH, PRIEST SS, DUDA LE, CARSON CC, HENDRICKS JD \& ROBINSON LC. 1988. Thermal regime of the State 2-14 well, Salton Sea Scientific Drilling Project. Jour. Geoph. Res., 93 (B11): 12995-13004.

SHEN P \& BECK AE. 1986. Stabilization of bottom hole temperature unith finite circulation time and fluid flow. Geoph. Jour. (Royal Astr. Soc.) 86: 63-90.

VACQUIER V. 1984. Oil fields - a source of heat flow data. Tectonophysics, 103: 81-98.

\section{NOTAS SOBRE OS AUTORES}

Anderson Gusmão Cavalcante é bacharel em Geofísica pela Universidade Federal da Bahia. Atualmente trabalha como geofísico na Compagnie Generale de Geophisique, CGG. É filiado à SBGf e à SEG.

Roberto Max de Argollo é doutor em Ciências, área Geofísica, pela Universidade Federal da Bahia, mestre em Oceanografia pela Universidade de Rhode Island, Estados Unidos e bacharel em Física pela então Universidade do Brasil. É professor titular do Instituto de Física e pesquisador do Centro de Pesquisa em Geofísica e Geologia, ambos da UFBa. Coordena o projeto Geoterm, financiado pela Finep em parceria com a Petrobras, para estudos geotérmicos de bacias sedimentares. Suas áreas de interesse são fluxo térmico, calor radiogênico e cronologia de sedimentos marinhos usando chumbo-210 e césio-137. É filiado à SBPC, à SBGf e à SBF.

Humberto da Silva Carvalho é doutor em Ciências, área Geofísica, mestre em Geofísica e bacharel em Física pela Universidade Federal da Bahia, É consultor do projeto Geoterm, financiado pela Finep em parceria com a Petrobras, para estudos geotérmicos de bacias sedimentares, que é desenvolvido no Instituto de Física/Centro de Pesquisa em Geofísica e Geologia da UFBa. Suas áreas de interesse são geotermia e fluxo térmico. É filiado à SBGf. 